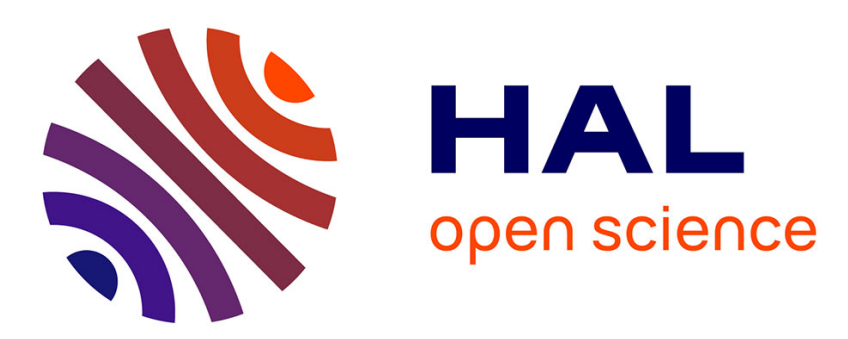

\title{
A time domain CLEAN approach for the identification of acoustic moving sources
}

Rémi Cousson, Quentin Leclere, Marie-Agnès Pallas, Michel Berengier

\section{To cite this version:}

Rémi Cousson, Quentin Leclere, Marie-Agnès Pallas, Michel Berengier. A time domain CLEAN approach for the identification of acoustic moving sources. Journal of Sound and Vibration, 2019, 443, pp.47-62. 10.1016/j.jsv.2018.11.026 . hal-01950898

\section{HAL Id: hal-01950898 \\ https://hal.science/hal-01950898}

Submitted on 11 Dec 2018

HAL is a multi-disciplinary open access archive for the deposit and dissemination of scientific research documents, whether they are published or not. The documents may come from teaching and research institutions in France or abroad, or from public or private research centers.
L'archive ouverte pluridisciplinaire HAL, est destinée au dépôt et à la diffusion de documents scientifiques de niveau recherche, publiés ou non, émanant des établissements d'enseignement et de recherche français ou étrangers, des laboratoires publics ou privés. 


\title{
A time domain CLEAN approach for the identification of acoustic moving sources
}

\author{
R. Cousson ${ }^{\mathrm{a}, \mathrm{b}}$, Q. Leclère $^{\mathrm{b}}$, M.-A. Pallas ${ }^{\mathrm{a}}$, M. Bérengier ${ }^{\mathrm{c}}$ \\ ${ }^{a}$ Univ Lyon, IFSTTAR, CEREMA, UMRAE, F-69675, Lyon, France \\ ${ }^{b}$ Univ Lyon, INSA-Lyon, Laboratoire Vibrations Acoustique, F-69621 Villeurbanne, France \\ ${ }^{c}$ IFSTTAR, CEREMA, UMRAE, F-44344 Bouguenais, France
}

\begin{abstract}
The issue of acoustic source identification has been widely explored in static or quasi-static source contexts, more recently for moving sources, in a wide range of applications. In the case of a passing-by vehicle, the beamforming method is a reference having well-known limitations that deconvolution methods try to overcome. Mostly developed in static source situations at the beginning, some recent deconvolution extensions to moving sources have been proposed in the transportation field, mainly in air transportation and underwater acoustics. In the present investigation, a new approach - called CLEANT for CLEAN-Time domain - is proposed and numerically tested with parameters fitted to the road vehicle context. Inspired by the CLEAN algorithm used to solve deconvolution problems, it is performed exclusively in the time domain and takes advantage of the source signal reconstruction available from the beamforming procedure. Its performance is assessed at several different speeds, source-to-array distances and with various additional noise levels, as well as its robustness regarding an uncertainty on some input parameters. The proposed approach is compared to a reference method using performance indicators. The simulations show better localization and quantification with CLEANT as compared to the other method tested in this road transportation context. Laboratory measurements in scaled pass-by conditions have been conducted and the results are presented and confirm the improvement brought by CLEANT.
\end{abstract}

Keywords: acoustics, acoustic imaging, source identification, beamforming, source separation, moving sources, road vehicles

\section{List of Abbreviations and Symbols}

Following symbols are used in the present work:

$\left[X_{F}, Y_{F}, Z_{F}\right]$ Focus point coordinates in the moving coordinate system

$\left[x_{m}, y_{m}, z_{m}\right] m^{\text {th }}$ microphone coordinates in the fixed observation coordinate system

$\left[X_{s}, Y_{s}, Z_{s}\right]$ Source point coordinates in the moving coordinate system

$\left[x_{s}, y_{s}, z_{s}\right]$ Source point coordinates in the fixed observation coordinate system

$\mathcal{T} \quad$ Recording time interval

$\gamma \quad$ CLEANT loop gain

$\Gamma^{(i)}(t, F)$ CLEANT clean "map" at iteration $i$

$\hat{F} \quad$ Focus point corresponding to the signal with maximum energy in the dirty "map"

$\Phi^{(i)}(t, F)$ CLEANT dirty "map" at iteration $i$

$\mathbf{B}(f) \quad$ Vector of quadratic beamforming outputs at the frequency $f$ 
$\mathbf{C}(f) \quad$ Cross spectral matrix of the microphone signals at the frequency $f$

$\mathbf{H}(f) \quad$ Energetic transfer function matrix at the frequency $f$

$\mathbf{S}(f) \quad$ Vector of quadratic source strengths at the frequency $f$

$\mathbf{w}_{i}(f) \quad$ Steering vector associated to the $i^{\text {th }}$ source at the frequency $f$

$\theta_{F m}(t)$ Angle between the source movement direction and the \{focus point $F$ - sensor $m$ \} direction at the emission time $t$

$\Theta_{s m}(t)$ Propagation angle between the source movement direction and the \{source $s$ - sensor $\left.m\right\}$ at time $t$

$\theta_{s m}(t) \quad$ Angle between the source movement direction and the \{source $s$ - sensor $m$ \} direction at time $t$

$b\left(t, F,\left\{p_{m}\right\}\right)$ Beamforming output at the focus point $F$, at emission time $t$ using the microphone signals $p$

$c \quad$ Speed of sound

F $\quad$ Focus point in the moving source-grid coordinate system

$K_{F m}(t)$ Beamforming normalization term

$M \quad$ Mach number

$m \quad$ Microphone index

$N_{F} \quad$ Total number of focus points on the source-grid

$N_{m} \quad$ Total number of microphones

$p_{m}(t) \quad$ Time signal on sensor $m$

$p_{m}^{\mathrm{res}}(t) \quad$ Residual signal on sensor $m$ after withdrawing the contribution of the dominant source(s)

$q(t) \quad$ Mass flow function of the source

$r_{F m}(t)$ Distance between the focus point $F$ and sensor $m$ at the emission time $t$

$R_{s m}(t)$ Propagation distance between the source point $s$ and sensor $m$ at time $t$

$r_{s m}(t) \quad$ Distance between the source point $s$ and sensor $m$ at time $t$

$s \quad$ Moving source point

$t_{s} \quad$ Emission time

$V \quad$ Constant vehicle speed

$W(t) \quad$ Apodization time window centered on the monitoring area

$w_{m} \quad$ Microphone weighting 


\section{Introduction}

Urban population is exposed daily to noise pollution, a great part of it being generated by road traffic. In order to reduce this nuisance, beside actions on infrastructure or planning, the acoustical optimization of road vehicles is a key lever. This requires improved knowledge of the acoustic behavior of vehicles. The identification of the vehicle noise sources in real driving conditions provides an objective diagnosis either for vehicle design or for the development of realistic prediction models.

Beamforming is a well-known technique which can be adapted to the localization of moving sources. Basically for static sources, it consists in making a directional filter by post-processing the signals received by an array of microphones. The process can be implemented either in time or in the frequency domain [1]. In the time domain "delay and sum" beamforming — the signals received by the microphone array are delayed, weighted and averaged. In the frequency domain, this operation is performed by the multiplication of the microphone cross-spectral matrix by "steering vectors". The calculation of the delays and steering vectors depends on the spatial point the array is focused on. Both delays and steering vectors are determined according to a model of source and propagation adapted to the measurement context. Unfortunately, the beamforming method has well known limitations, explained for instance in [2], particularly a poor resolution in low frequency and a difficulty to provide a good level estimation in the case of multiple close sources or extended sources. To overcome these issues, deconvolution methods have been developed to improve the localization and the estimation of the power level radiated by static sources [3][4].

Developments have been made to adapt beamforming to moving sources in the time domain by steering the focus point with the moving point-source, by means of time-varying delays [5]. Such a technique has been used in a large variety of context and is naturally suited to pass-by situations [6], for either road vehicles [7, 8, 9], aircraft $[10,11$, $12,13]$, trains [5, 14] or ships [15, 16]. Several deconvolution approaches have been adapted to the moving source problem. Fleury and Bulté [17] proposed a solution which was tested in simulation and real experiment in the case of an aircraft flying over. A variant in the context of underwater acoustics was implemented in the case of a ship, studied by Oudompheng [16] and Lamotte et al. [18], involving far lower vehicle speeds. The performance of this method has been numerically assessed in the case of road vehicle pass-by conditions and with an academic experimental set-up in [19], and compared to a reference method. This reference was a conventional moving source beamforming, followed by a deconvolution performed with a point spread function (PSF) corresponding to the static case. Although this comparison was not optimal, since the deconvolution for mobile sources was implemented in a degraded way, the method proposed by Fleury and Bulté showed an improvement and promising results in a road context. However some limitations were also pointed out in terms of separation power and frequency range.

The present paper proposes a new development, exclusively performed in the time domain, that takes advantage of the fact that time domain beamforming actually reconstructs the signal emitted from the focus point. The nonstationary nature of a pass-by measurement makes it interesting to operate in time domain, especially to take into account the Doppler effect. In section 2, the method is described and discussed. Then, simulations are presented in section 3: indicators to evaluate the performance of the method are defined and used to compare it to the approach by Fleury and Bulté using a moving source adapted point spread function [17] (noted MSA-PSF). Section 4 is dedicated to an academic experimental set-up designed to test and compare the methods on moving sources in scaled vehicle pass-by conditions. At last there is a discussion about the performance of the new method in both simulation and experimental conditions.

\section{Problem formulation}

\subsection{Identification of static sources}

\subsubsection{Conventional beamforming in frequency domain (CBF-FD)}

The common method to identify static sources with a phased array of microphones is conventional beamforming. It can be performed either in time-domain or frequency-domain. In either case, the location and the power of sources in a scan area are estimated thanks to the signals received by an array of sensors. In the frequency-domain, the power estimate from the $i^{\text {th }}$ point in the sampled scan area is, at each frequency $f$ :

$$
B_{i}(f)=\mathbf{w}_{i}^{*}(f) \mathbf{C}(f) \mathbf{w}_{i}(f)
$$


where $\mathbf{w}_{i}$ is a steering vector depending on the source and propagation models, $*$ denotes the complex conjugate transpose and $\mathbf{C}$ is the cross-spectral matrix of microphone signals.

Conventional beamforming works as a spatial filter but has a poor resolution in low frequency and is not very accurate to quantify the power of extended sources or in a multi-source environment. Deconvolution methods have been developed to overcome those limitations and are briefly presented in section 2.1.2.

\subsubsection{Deconvolution}

Most deconvolution methods are applied to a beamforming output that has been performed in the frequency domain. For time-invariant conditions and decorrelated sources, the deconvolution consists in inversing the following system:

$$
\mathbf{B}(f)=\mathbf{H}(f) \mathbf{S}(f) \quad \text { with } \quad \mathbf{S} \geq 0
$$

where $\mathbf{B}$ is the vector of quadratic beamforming outputs for the set of focus points on the grid representing the sampled scan area, $\mathbf{H}$ is an energetic transfer function matrix and $\mathbf{S}$ is a vector representing the unknown quadratic source strengths. $\mathbf{H}$ depends on the model of sources and propagation chosen according to the physical context. When considering point sources, the columns of $\mathbf{H}$ are called Point Spread Functions (PSF).

Various algorithms are available in the literature and can be used to find a solution to Eq. (2), for instance DAMAS, CLEAN or NNLS (non-negative least square). DAMAS was proposed by Brooks and Humphreys [20], then improved in terms of calculation time by Dougherty [21]. It aims to reconstruct the correct source level distribution by solving the system of equations (2) with a modified Gauss-Seidel algorithm, enforcing the non-negativity condition. CLEAN was initially developed in astronomy [4] and later applied to acoustics [22, 23]. In this algorithm, the highest peak value is searched among the source powers found in the beamforming output. The position and amplitude of the source corresponding to this maximum value are updated in a so-called "clean" map. Then the source image through the PSF is subtracted from the original map. The process is reiterated until a stopping criterion or a maximum number of iterations is reached. CLEAN has then been improved by Sijtsma [24] with CLEAN-SC which takes into account the spatial coherence of sources.

While deconvolution can be very effective for static sources, its application to moving sources is more challenging. The non-stationary nature of the moving source problem is troublesome, since the transformation between the signals emitted by the sources and the signals received by the microphones can no longer be written as a convolution and thus, as a simple product in frequency-domain [16,17]. This problem must be addressed in a different way, as it is presented in section 2.2.1.

\subsection{Identification of moving sources}

\subsubsection{Swept-focus point beamforming}

Let us consider a vehicle, moving at a constant speed $V$, and an array of $N_{m}$ microphones. To simplify the equations, we assume the vehicle has a linear movement in the $\mathrm{x}$-axis direction: $V=\left[V_{x}, 0,0\right]$. A source $s$ attached to the vehicle at position $\left[X_{s}, Y_{s}, Z_{s}\right]$ in the moving coordinate system is located, in the fixed observation coordinate system, at:

$$
x_{s}(t)=V_{x} t+X_{s} \quad y_{s}(t)=Y_{s} \quad z_{s}(t)=Z_{s}
$$

and the origin of the moving coordinate system is located at $x_{s}=0$ at the time $t=0$.

The signal emitted by the source $s$ at the time $t_{s}$ is received by sensor $m$ at position $\left[x_{m}, y_{m}, z_{m}\right]$ at the reception time $t=t_{s}+\frac{r_{s m}\left(t_{s}\right)}{c}$, where $r_{s m}(t)$ is the distance between the position of source $s$ and sensor $m$, and $c$ is the speed of sound. The angle between the source movement direction and the source-sensor direction is $\theta_{s m}(t)$ (Fig. 1).

According to Morse \& Ingard[25], the signal emitted by a monopole and received by sensor $m$ in this situation is:

$$
p_{m}(t)=\frac{Q\left(t-\frac{R_{s m}(t)}{c}\right)}{R_{s m}(t)\left(1-M \cos \Theta_{s m}(t)\right)^{2}}, R_{s m}(t)=r_{s m}\left(t_{s}\right), \Theta_{s m}(t)=\theta_{s m}\left(t_{s}\right)
$$


where $Q(t)=\frac{q^{\prime}(t)}{4 \pi}, q^{\prime}(t)$ is the derivative of the source mass flow and $M$ is the Mach number $\left(M=\frac{V_{x}}{c}\right) . R_{s m}(t)$ and $\Theta_{s m}(t)$ are explicit expressions of the propagation distance $r_{s m}\left(t_{s}\right)$ and the propagation angle $\theta_{s m}\left(t_{s}\right)$ respectively, as a function of the reception time $t$. Their explicit formulations are also given by Morse $\&$ Ingard:

$$
\begin{aligned}
& R_{s m}(t)=\frac{M\left(x_{m}-\left(X_{s}+V t\right)\right)+R_{s m}^{\prime}(t)}{1-M^{2}} \\
& R_{s m}^{\prime}(t)=\sqrt{\left(x_{m}-\left(X_{s}+V t\right)\right)^{2}+\left(1-M^{2}\right)\left[\left(Y_{s}-y_{m}\right)^{2}+\left(Z_{s}-z_{m}\right)^{2}\right]} \\
& \cos \Theta_{s m}(t)=\frac{R_{s m}(t)-R_{s m}^{\prime}(t)}{M R_{s m}(t)}
\end{aligned}
$$

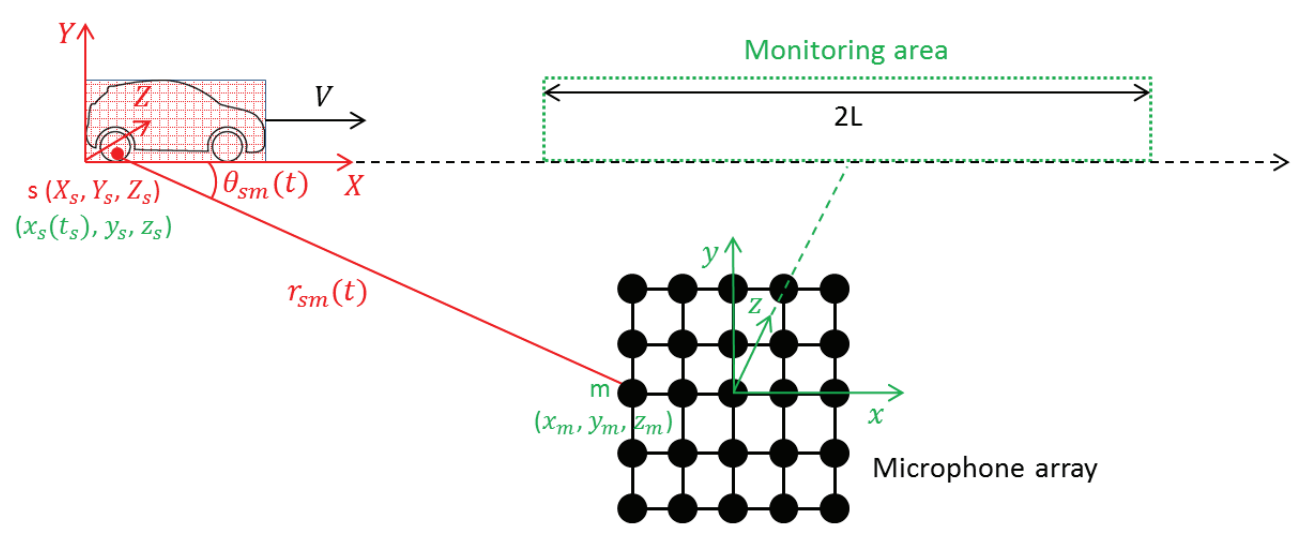

Figure 1: Pass-by configuration in the fixed (green) and moving (red) coordinate systems.

The focusing operation consists in recovering a signal $Q_{F}(t)$ at a focus point $F$ (position $\left[X_{F}, Y_{F}, Z_{F}\right]$ in the moving coordinate system) from measured acoustic pressures $p_{m}(t)$. In this situation we need to change the time basis of Eq. (4):

$$
p_{m}\left(t+\frac{r_{F m}(t)}{c}\right)=\frac{Q_{F}(t)}{r_{F m}(t)\left(1-M \cos \theta_{F m}(t)\right)^{2}}
$$

where $r_{F m}(t)$ and $\theta_{F m}(t)$ are simply given by

$$
\begin{aligned}
& r_{F m}(t)=\sqrt{\left(x_{m}-\left(X_{F}+V t\right)\right)^{2}+\left(y_{m}-Y_{F}\right)^{2}+\left(z_{m}-Z_{F}\right)^{2}} \\
& \cos \theta_{F m}(t)=\frac{x_{m}-\left(X_{F}+V t\right)}{r_{F m}(t)}
\end{aligned}
$$

Beamforming consists in focusing microphones signals $\left\{p_{m}\right\}\left(m \in\left[1 \ldots N_{m}\right]\right)$ at a point $F$ on the source-grid constituted of $N_{F}$ points, which samples the area scanned on the vehicle. The beamforming output at this point is, in the case of moving sources:

$$
b\left(t, F,\left\{p_{m}\right\}\right)=\frac{W\left(t+\frac{X_{F}}{V}\right)}{K_{F m}(t)} \sum_{m=1}^{N_{m}} w_{m} \frac{p_{m}\left(t+\frac{r_{F m}(t)}{c}\right)}{r_{F m}(t)\left(1-M \cos \theta_{F m}(t)\right)^{2}},
$$

where $w_{m}$ are spatial weighting coefficients and $K_{F m}(t)$ is a normalization term:

$$
K_{F m}(t)=\sum_{m=1}^{N_{m}} \frac{w_{m}}{\left[r_{F m}(t)\left(1-M \cos \theta_{F m}(t)\right)^{2}\right]^{2}}
$$


and where $W(t)$ is a centered apodization window (of Tukey type for instance) which is null for all $|t|>L / V$ where $L$ is half the length of the monitoring area (Fig 1).

$b\left(t, F,\left\{p_{m}\right\}\right)$ can be considered as an estimate of the time signal $Q_{F}(t)$ emitted from the moving focus point $F$ during its transit in the monitoring area (Fig. 1). This area is defined in order to avoid an unacceptable broadening of the beamforming response main lobe when focusing at high angles from the normal to the array. The focus point moves along with the vehicle, so knowledge of the vehicle kinematics is essential. Beamforming, implemented as described in this section, will be mentioned as CBF-TD (for Conventional Beamforming in Time Domain) from this point.

\subsubsection{Deconvolution methods adapted to moving sources - MSA-PSF}

Some authors tried to tackle the problem of applying deconvolution algorithms, which operate in frequencydomain, to moving sources situations. A first approach consists in using a time-domain moving source beamforming and then in doing the deconvolution with a modified PSF which takes into account the Doppler frequency shift caused by the source motion [26]. Even though this approach gives promising results, it is not totally satisfying, as the PSF is integrated over a frequency band which becomes larger as the frequency shift becomes greater. This method is operational for tone sources.

While the former method tries a hybrid approach between a time-domain beamforming and a frequency-domain deconvolution, Fleury and Bulté proposed a solution exclusively performed in the frequency domain to identify moving sources [17]. Even though its authors did not give it a specific name, we chose to call it Moving Source Adapted PSF (MSA-PSF) to conveniently mention it throughout this paper.

It consists in splitting the full time problem into several snapshots. Within each snapshot, the movement is considered as quasi-static, and a frequency domain beamforming (CBF-FD) is applied, with a correction that takes into account the Doppler effect in both frequency and signal amplitude. A deconvolution is then performed on the averaged beamforming outputs and PSF over all the snapshots. The main hypothesis for this method are:

- The sources are uncorrelated from one another and for a single source from one frequency to another.

- The sources are monopoles, linearly moving at a constant speed $V$.

- The quasi-static assumption is valid if the following condition is satisfied:

$$
V T_{\text {snap }} \ll D
$$

which means the displacement of the sources within the duration of a snapshot is small in regards of the sourceto-microphone distance. Under the previous assumptions, the source-to-microphone distance, the angle and the dopplerized frequency $f_{\text {dopp }}=\frac{f}{1-M \cos \theta}$ can be considered as constant within a snapshot and their values are taken at the center of the snapshot.

- The random variables are ergodic. Statistical quantities can be calculated by averaging over the snapshots.

- The differences between the dopplerized frequencies received on a microphone from every source-point considered are smaller than the frequency sample rate.

Even though this method provides good results in aeroacoustic applications, the road vehicle context has different characteristics, making the possible application of MSA-PSF uncertain in this case. Indeed, it differs from the cases studied in $[16,17,18]$ in the vehicle speed and distance from the microphone array, ultimately resulting in a difference in the pass-by duration. Contrary to [17] for instance, the Doppler frequency shift varies more rapidly during a snapshot because the vehicle is closer to the array, and the microphone signals cannot be considered as strictly quasistationary (because of the non-constant Doppler shift within a snapshot). The method's performance in this context was assessed in [19], and though it showed quite satisfying results, some limitations were pointed out, especially in terms of frequency range. This motivated the development of another approach. 


\subsubsection{CLEANT procedure}

CLEANT is a method which is inspired by the deconvolution algorithm CLEAN, but performed in time-domain. A similar approach called "acoustic eraser" was proposed by Döbler and Schröder [27, 28] for static sources with the aim of increasing the beamforming map dynamic by iteratively removing dominant sources in time-domain. This allowed to gain access to weaker sources which were masked by the main ones and their spatial extent in the map. They later proposed to create an acoustic map with a better resolution using this technique, making a clear parallel with both CLEAN and the high dynamic range (HDR) process used in photography, using this acronym for their method [29]. Our own approach can be seen as an extension of this work, by including a gain factor in the same way as CLEAN to increase its robustness, and by considering the application to moving sources while the acoustic eraser and HDR were only used in static context.

CLEANT is an iterative deconvolution approach which operates in the time domain. Using conventional moving source beamforming, it gradually subtracts the source time contributions from the microphone signals. The "dirty map" and "clean map" specific to the CLEAN method [4] are of a different nature in this CLEANT procedure: they are arrays of source time signals associated with the main source locations. The term "map", although inappropriate, is used in the following for these arrays so as to highlight the parallels with CLEAN.

The beamforming mentioned in the algorithm corresponds to the moving source beamforming as described in 2.2.1. The output is composed of the signals $b\left(t, F,\left\{p_{m}\right\}\right)$ reconstructed for each point $F$ of the source-grid during the time interval $T_{F}$, the signal outside of this interval being considered as zero.

The algorithm performs the following steps:

1. Initialization of the dirty "map" $\Phi$ and the clean "map" $\Gamma$ at iteration $i=0$ :

$$
\begin{aligned}
\Phi^{(0)}(t, F) & =b\left(t, F,\left\{p_{m}\right\}\right) \quad \forall F \in \llbracket 1 ; N_{F} \rrbracket \\
\Gamma^{(0)}(t, F) & =0 \\
p_{m}^{\text {res }(0)}(t) & =p_{m}(t)
\end{aligned}
$$

where $p_{m}^{\text {res }}$ is the signal on microphone $m$ cleaned from the dominant source time signal contribution, initialized with the recorded microphone signal and updated in step 3.

2. At each iteration $\mathrm{i}(i \geq 1)$, detection of the focus point $\hat{F}$ maximizing the energy of the dirty "map" signals:

$$
\hat{F}=\underset{F}{\operatorname{argmax}}\left(\int_{\mathcal{T}}\left|\Phi^{(i-1)}(t, F)\right|^{2} d t\right)
$$

3. A part of the dominant source contribution is removed from the microphone signals:

$$
p_{m}^{\mathrm{res}(i)}\left(t+\frac{r_{\hat{F} m}}{c}\right)=p_{m}^{\mathrm{res}(i-1)}\left(t+\frac{r_{\hat{F} m}}{c}\right)-\gamma \frac{\Phi^{(i-1)}(t, \hat{F})}{r_{\hat{F} m}(t)\left(1-M \cos \theta_{\hat{F} m}(t)\right)^{2}} \quad \forall m \in \llbracket 1 ; N_{m} \rrbracket
$$

where $\gamma \in[0,1]$ is the CLEANT loop gain. The resampling to get the emission time from the reception time here is carried out by a spline interpolation, with the Matlab interp1 function.

4. Clean "map" is updated by adding a part of the detected dominant source signal:

$$
\Gamma^{(i)}(t, \hat{F})=\Gamma^{(i-1)}(t, \hat{F})+\gamma \Phi^{(i-1)}(t, \hat{F})
$$

5. Dirty "map" is updated by computing the beamforming output using the residual microphone signals:

$$
\Phi^{(i)}(t, F)=b\left(t, F,\left\{p_{m}^{\mathrm{res}(i)}\right\}\right) \quad \forall F \in \llbracket 1 ; N_{F} \rrbracket
$$

6. A new iteration starts back at step 2 unless one of the stopping criteria is reached.

The stopping criteria are defined as follows:

- At each iteration, the total amount of energy left in the dirty "map" should decrease. However, when all the significant sources have been removed, only noise is left and this energy can increase from this point: the algorithm can stop when the energy at an iteration is higher than at the previous one. 
- A maximum number of iteration can be set by the user: depending on the loop gain chosen and the possible knowledge of the number of sources expected, the user can shorten the duration of the method without waiting for the first criterion to be reached.

The CLEANT loop gain $\gamma \in[0,1]$ is chosen to remove only a portion of the dominant source estimate found at each iteration. Indeed, the estimate of the source at this location is likely to include contributions from the beamforming sidelobes of nearby sources. For an optimum robustness of the algorithm, one should choose a quite small value for $\gamma$. However, the smaller the loop factor, the longer the algorithm. The choice for $\gamma$ depends on the situation and different values should be tested by the user to find the best compromise between robustness and speed.

In the end, all the non null reconstructed source time signals are stored, as well as the associated positions, and become available for further analysis, such as a power spectral density estimate for instance. Then, using the clean "map" $\Gamma(t, F)$, we can create a deconvoluted display of the source locations and of their levels on the source-grid, at any frequency.

\subsection{Advantages and drawbacks of the CLEANT approach}

The main concern for this method is that it can be time consuming. Since every step is carried out in time domain, the moving source beamforming step as well as the moving source repropagation represent the main time expense, since they involve interpolation. Depending on the situation (experimental conditions, signal-to-noise ratio, number of sources, etc), the algorithm duration can be either negligible or on the contrary a serious drawback. An illustration of the time consumption by the three methods tested in section 4 is presented along with the experimental results in section 4.

What may be lost in time consumption is gained in accuracy. In the case of moving sources, the Doppler effect can be effectively taken into account, allowing to apply the method to high speed moving sources. As presented here, CLEANT is a wideband procedure, but the recorded signals could be filtered after the beamforming stage to aim for specific frequency bands, which can also improve the result in case of close sources with well separated frequency contents.

Another advantage is that all the reconstructed main source time signals are available. It is then possible to carry out other types of analysis requiring time signals: referenced source separation [30], fault detection [31], classification [32], auralization [33], etc.

\section{Validation of the method with synthetic data}

A series of simulations have been conducted to test the performance and robustness of the CLEANT method in the case of road transportation, covering a range of situations that are common in pass-by vehicle measurement. The results are compared with the method MSA-PSF (moving source adapted point spread function) described in [17] and [19], and briefly presented in section 2.2.2.

\subsection{Simulation parameters}

\subsubsection{Configurations and parameters}

Without loss of generality, the source area is assumed as one dimensional in the simulations. A four meter long vehicle is considered. The source-grid abscissa extends from $-2 \mathrm{~m}$ to $+2 \mathrm{~m}$ with a $5 \mathrm{~cm}$ step. The sampling frequency is $f_{\text {sample }}=12 \mathrm{kHz}$ and the range of frequencies studied spans from $400 \mathrm{~Hz}$ to $5 \mathrm{kHz}$. The sampling frequency is intentionally chosen at a low rate to limit processing times and test the methods within this constraint. The array is a $3.9 \mathrm{~m}$ long line array composed of 35 microphones. The sensor interspace regularly increases from the center towards the end of the array, with a $\log 2$ factor: from $3 \mathrm{~cm}$ near the center up to $15 \mathrm{~cm}$ near the end. At $5 \mathrm{kHz}$, the wavelength is $\lambda=6.8 \mathrm{~cm}$, and $\lambda / 2=3.4 \mathrm{~cm}$, still providing an unaliased array pattern over the monitoring area. In order to study the localization and quantification performance of the method, a unique source is considered, located at the center of the moving source-grid $\left(X_{s}=0\right)$. The source signal is a white gaussian noise. These geometrical parameters are illustrated in Fig. 2.

The varying configuration parameters are:

- The speed $V$ of the vehicle. 


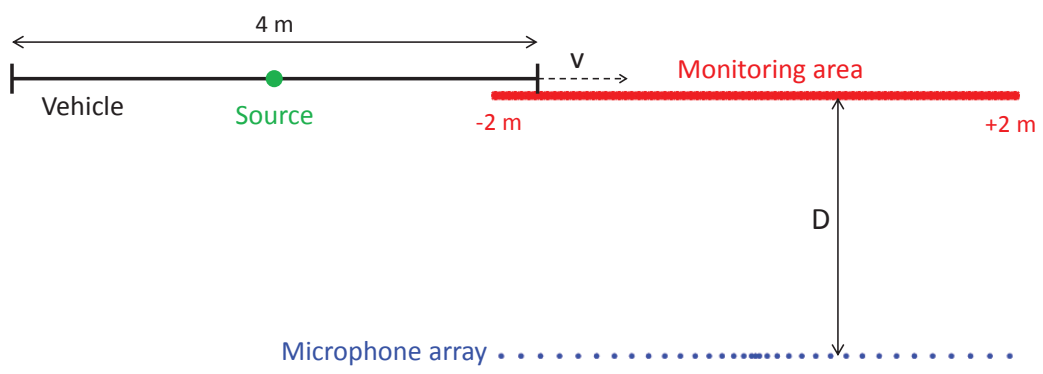

Figure 2: Simulation configuration.

- The distance $D$ between the plane of the moving vehicle and the array plane.

- The signal-to-noise ratio (SNR), considering an additive white noise on each microphone, uncorrelated to the source signal and from one sensor to the other.

The duration of the snapshots in the MSA-PSF method is fixed to $20 \mathrm{~ms}\left(\Delta_{f}=50 \mathrm{~Hz}\right)$ and is chosen to satisfy the condition expressed in Eq. (7) for the highest vehicle speed while having a reasonably good frequency accuracy. The total number of snapshots varies with the vehicle speed from 17 to 45 with $60 \%$ overlap in order to keep a monitoring area ranging from abscissa $-2 \mathrm{~m}$ to $+2 \mathrm{~m}$. In the CLEANT algorithm, the beamforming stage is applied on the same monitoring area $[-2 \mathrm{~m} ;+2 \mathrm{~m}]$.

The loop gain in the CLEANT algorithm is set to 0.7. In addition to the energy stopping criterion described in 2.2.3, a maximum number of iterations is defined and set to 20. All the configurations that have been tested are summarized in Table 1.

Table 1: Simulation parameter configurations: $V$ (speed of the vehicle), $D$ (source-to-array distance) and SNR (signal-to-noise ratio).

\begin{tabular}{cccc}
\hline Config. Name & $V\left(\mathbf{k m . h ^ { - 1 }}\right)$ & $D(\mathbf{m})$ & SNR $(\mathbf{d B})$ \\
\hline V30 & 30 & & \\
V50 & 50 & 4 & No noise \\
V70 & 70 & & \\
V90 & 90 & & \\
\hline D3 & & 3 & \\
D4 & 50 & 4 & No noise \\
D5 & & 5 & \\
N10 & & & 10 \\
N5 & 50 & 4 & 5 \\
N0 & & & 0 \\
\hline
\end{tabular}

\subsubsection{Performance indicators}

In order to assess the method performance in terms of source localization and quantification, a set of indicators has been defined. Indicators using only the deconvolution grid-point maximum have been discarded because of their poor quality, especially for source quantification due to picket fence effect of spatial sampling (improvement with regard to this indicator would require a finer spatial sampling rate). Indicators considering a slightly spread source area are more accurate and only them have been kept.

- Position error: This indicator evaluates the gap between the estimated and the actual synthesized source locations. The former is the abscissa of the center of gravity of the source area found after deconvolution, taking into account the two adjacent grid-points of the deconvolution maximum as well. 
- Quantification error: This indicator evaluates the gap between the estimated source level, calculated as the cumulated level of the 3-grid-point wide source area centered on the deconvolution maximum, and the actual synthesized source level.

Averaged values and standard deviation of each indicator are carried out with 200 outcomes of the same experiment for each configuration. Whereas the mean indicator value informs on biased estimates, the information on dispersion is important since, practically, there is only one realization in the real case of a vehicle pass-by. Thus, the lower the dispersion, the more reliable the estimate.

\subsection{Effect of parameters on the method performance}

\subsubsection{Speed parameter}

The effect of the source speed on the performance of the various methods is evaluated with an array distance of 4 meters and no additive noise on the microphones (Table 1).

The CLEANT source localization is excellent at any speed, since the mean error is zero in all cases (the curves are superposed) and the standard deviation is also zero (Fig. 3b). The results are better than with MSA-PSF, even though this one also gives quite satisfactory results with unbiased position estimates. Its standard deviation of the position error indicator stays quite small on most of the frequency range but slightly grows at low frequencies. We also observe that the standard deviation of the position error hardly varies with the vehicle speed. Thus, only one standard deviation curve is displayed on Fig. 3a, corresponding to the $V=50 \mathrm{~km} . \mathrm{h}^{-1}$ case.

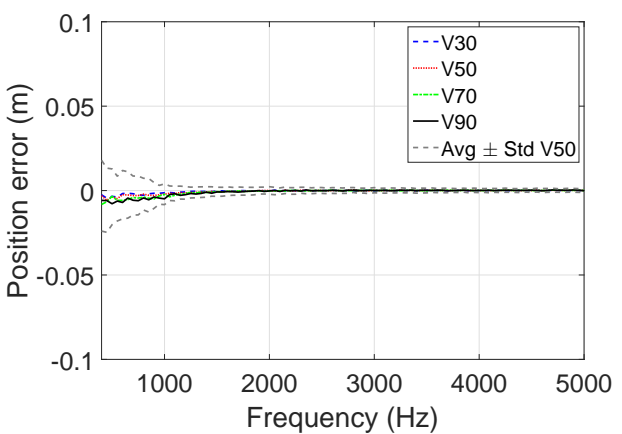

(a)

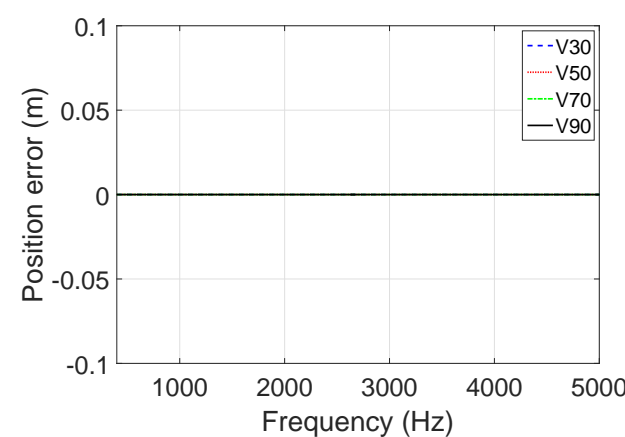

(b)

Figure 3: Effect of speed on the localization of a moving source - (a) MSA-PSF position error; (b) CLEANT position error - Colored lines: averaged error; Gray lines: averaged error \pm standard deviation at $50 \mathrm{~km} \cdot \mathrm{h}^{-1}$.

The source level estimation by CLEANT (Fig. 4b) is also very satisfactory within the frequency range studied. On the other hand, the quantification error increases with frequency for MSA-PSF (Fig. 4a). Still limited to $1 \mathrm{~dB}$ at $3.5 \mathrm{kHz}$, it gradually worsens with frequency and exceeds $4 \mathrm{~dB}$ at $5 \mathrm{kHz}$, which could be improved by increasing the sampling frequency. The dispersion of the results is strongly dependent on vehicle speed (Fig. 4a and 4d). CLEANT has a lower dispersion than MSA-PSF at low frequency (400-1500 Hz), not exceeding $1.5 \mathrm{~dB}$ in both cases.

\subsubsection{Distance parameter}

The effect of the source-to-array distance $D$ on the performance of the various methods is evaluated with a vehicle speed of $50 \mathrm{~km} \cdot \mathrm{h}^{-1}$ and no additive noise on the microphones (Table 1).

The comparison between the two methods regarding the distance parameter is quite similar to what was found with speed when considering the localization performance in both averaged values and dispersion of the position error indicator. Thus, the localization indicators are not represented here for the distance parameter.

However, the source level estimation behaves in the same way when the source-to-array distance varies as when the vehicle speed varies if considering the mean error (Fig. 5a and 5b) but not the dispersion (Fig. 5c). While the latter is strongly dependent on the vehicle speed, it doesn't depend on the source-to-array distances 3, 4 or $5 \mathrm{~m}$, for either method. Hence, only the standard deviation corresponding to the case $D=4 \mathrm{~m}$ is drawn (Fig. 5c). CLEANT makes 


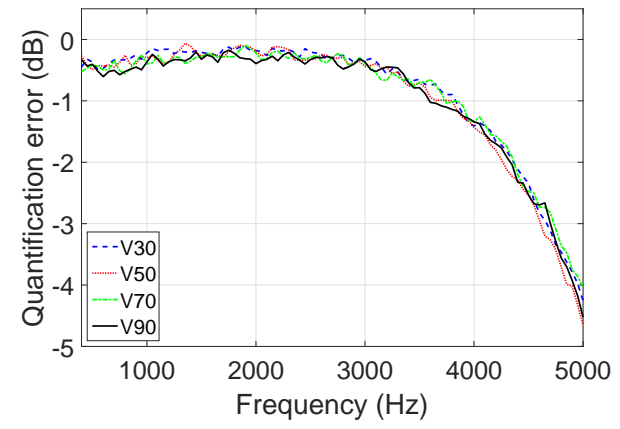

(a)

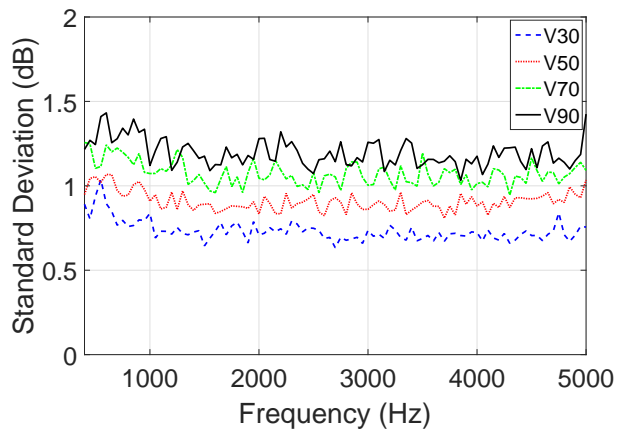

(c)

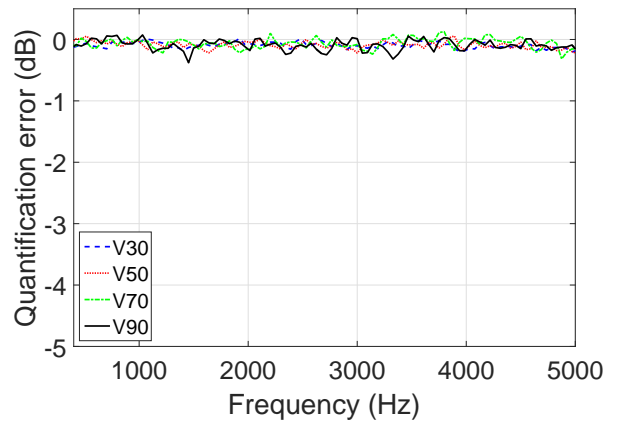

(b)

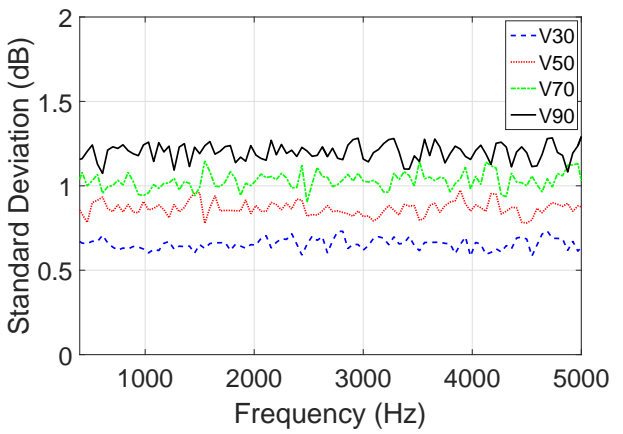

(d)

Figure 4: Effect of speed on the quantification of a moving source - (a)-(b) averaged quantification error of respectively MSA-PSF and CLEANT; (c)-(d) standard deviation of the quantification error of respectively MSA-PSF and CLEANT.

a slight difference at low frequency where the dispersion is lesser than with MSA-PSF, while the standard deviation becomes roughly the same over $1 \mathrm{kHz}$.

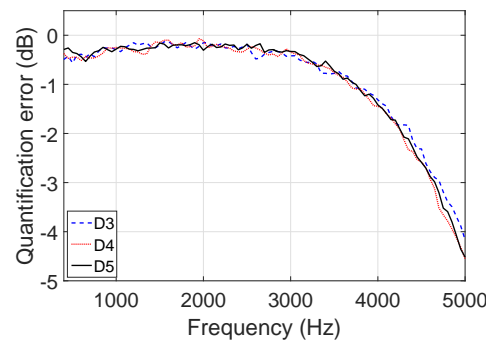

(a)

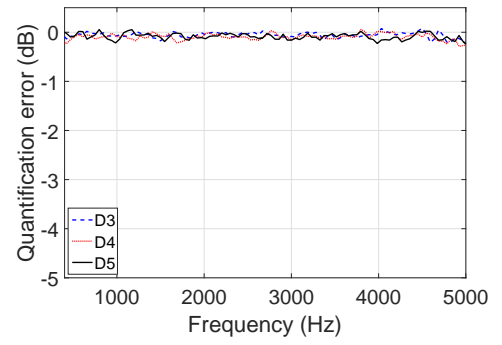

(b)

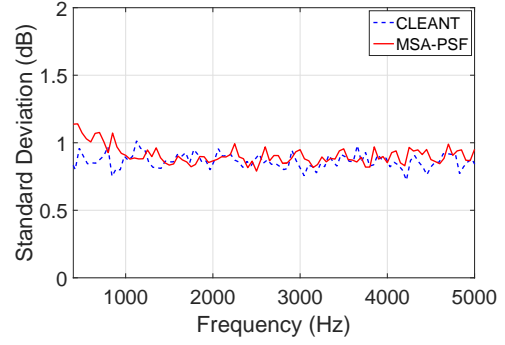

(c)

Figure 5: Effect of source-to-array distance on the quantification of a moving source - (a) averaged quantification error of MSA-PSF; (b) averaged quantification error of CLEANT; (c) standard deviation for $D=4 \mathrm{~m}$.

\subsubsection{Signal-to-noise ratio parameter}

The effect of an additive noise on the microphones, incoherent with the source signal and between sensors, on the performance of the various methods is evaluated with an array distance of 4 meters and a vehicle speed of $50 \mathrm{~km} . \mathrm{h}^{-1}$ (Table 1).

Considering the localization performance for various signal-to-noise ratios (SNR), CLEANT provides a clear improvement over MSA-PSF, particularly in terms of dispersion of the results. For conciseness, the figures are not 
represented since similar to Fig 3 .

However, there is a noticeable difference from the other two parameter situations regarding the quantification error. Whereas MSA-PSF is not very sensitive to SNR (Fig. 6a), CLEANT quantification performance depends on the presence of an additive noise (Fig. 6b) with a degradation of the mean source level (biased estimate) when SNR decreases, mostly at high frequency. This can be explained by the iterative nature of CLEANT which propagates noise at each iteration. The interpolation, occurring during the dedopplerization, can also be influenced by this additive noise. Thus, the method can be made less sensitive to noise both by adjusting the stopping criteria and by increasing the sampling frequency.

It is worth noting that except for the rather severe case of an SNR of $0 \mathrm{~dB}$, where CLEANT has no better performance than MSA-PSF, it still yields better results at an SNR of 5 or $10 \mathrm{~dB}$. Thus, this sensitivity to noise is a relative weakness of the method and CLEANT mostly remains an improvement compared to MSA-PSF.

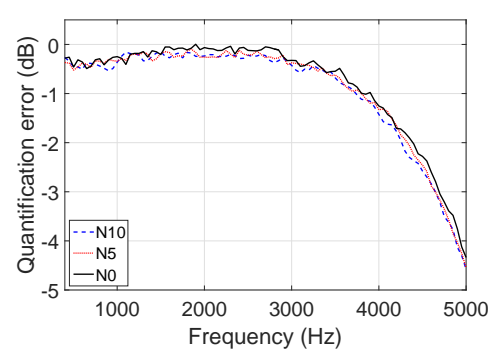

(a)

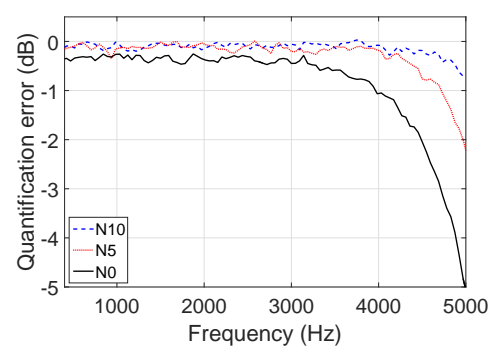

(b)

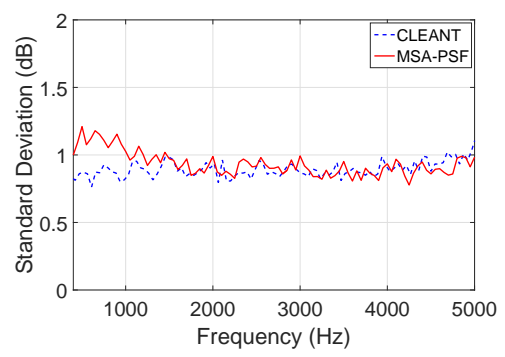

(c)

Figure 6: Effect of the SNR on the quantification of a moving source - (a) averaged quantification error of MSA-PSF; (b) averaged quantification error of CLEANT; (c) standard deviation for $\mathrm{SNR}=0 \mathrm{~dB}$.

The dispersion of the quantification results doesn't strongly depend on the value of the SNR for any of the two methods and only the standard deviation corresponding to the case SNR $=0 \mathrm{~dB}$ is represented (Fig. 6c). Despite its quantification bias being sensitive to additive noise, CLEANT standard deviation always remains under $1 \mathrm{~dB}$.

\subsection{Effect of an uncertainty in a parameter input on the method performance}

In this section, the performance of CLEANT is investigated in the case of an error on the input parameters, rendering an inaccurate knowledge of the actual configuration parameters. Regarding speed, a severe (5\% error) and a more likely (2\% error) scenarios have been considered, both either under- or overvalued. Regarding the sourcearray distance, the actual location of vehicle sources may not be strictly confined on the grid plane. Thus, CLEANT performance has been assessed when the source is actually located $20 \mathrm{~cm}$ nearer (resp. farther) than the grid plane.

In the study of both speed and distance error input, a vehicle driving at $50 \mathrm{~km} \cdot \mathrm{h}^{-1}$ at a $4 \mathrm{~m}$ distance from the array and without additional noise has been simulated.

\section{Speed input parameter}

It turns out that CLEANT is not severely impacted by an incorrect speed input in terms of the averaged values of the localization error (Fig. 7b), but is more so in terms of dispersion at high frequencies. On the other hand, MSAPSF localization performance drops noticeably, particularly at low frequency where the dispersion increases (Fig. 7a). For a better readability, only the dispersion corresponding to the worst dispersion case (the 5\% overvalued speed) is displayed in Fig. 7.

The source level estimation is also degraded for both methods, and the level error unsurprisingly increases with the speed input error, leading to a systematic underestimation of the source contribution (Fig. 8). The 5\% error scenarios show how they are dependent on the knowledge of the vehicle kinematics. While they are roughly equivalent from $400 \mathrm{~Hz}$ to $3000 \mathrm{~Hz}$, CLEANT is actually the most robust at higher frequencies, thanks to its loop gain $\gamma$. Regarding the dispersion of the quantification results, the standard deviation stays approximately constant around $1 \mathrm{~dB}$, except under $1500 \mathrm{~Hz}$ for MSA-PSF where it increases without reaching $1.5 \mathrm{~dB}$. Those figures are not displayed here for concision's sake. 


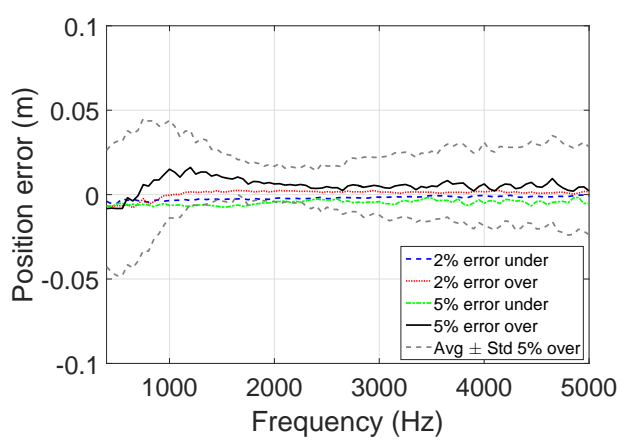

(a)

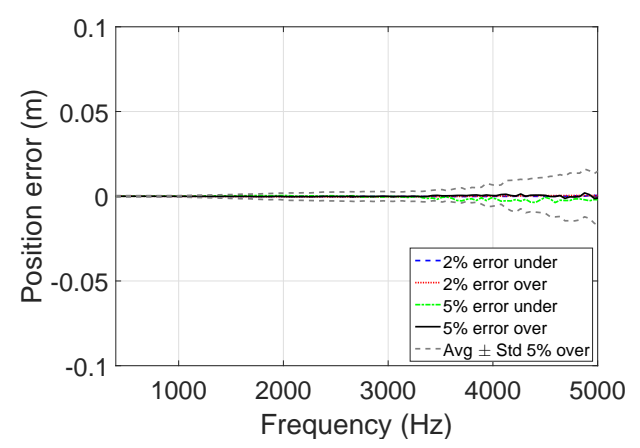

(b)

Figure 7: Error on the localization of a moving source with a wrong vehicle speed input - (a) MSA-PSF position error; (b) CLEANT position error - Colored lines: averaged error; Gray lines: averaged error \pm standard deviation for a $5 \%$ overvalued speed.

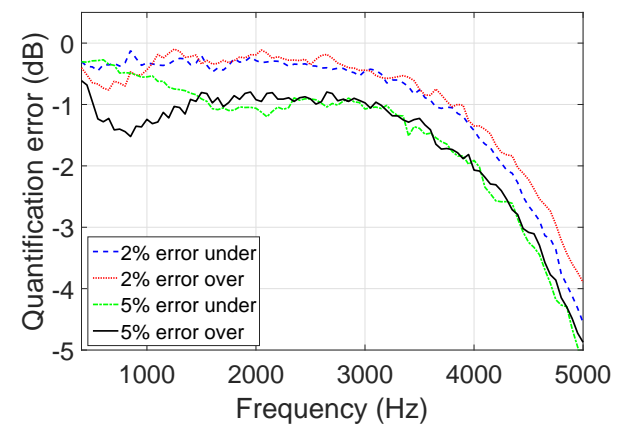

(a)

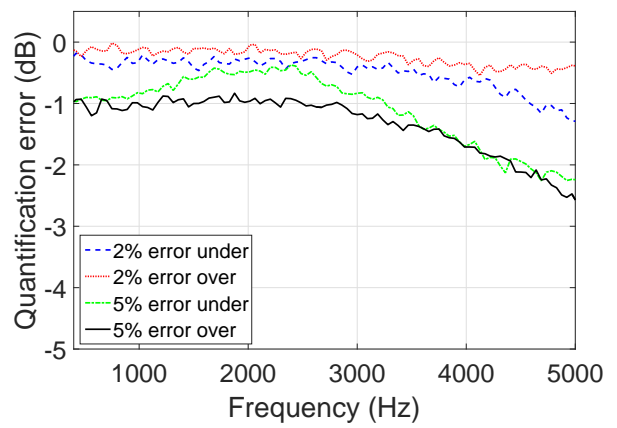

(b)

Figure 8: Averaged error on the quantification of a moving source with a wrong vehicle speed input - (a) MSA-PSF quantification error; (b) CLEANT quantification error.

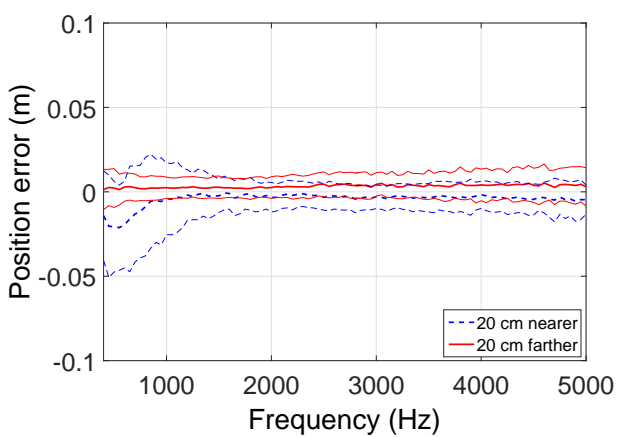

(a)

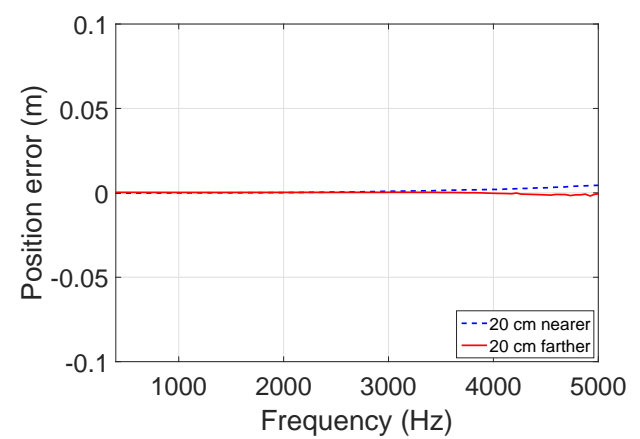

(b)

Figure 9: Error on the localization of a moving source with a wrong source-to-array distance - (a) MSA-PSF position error; (b) CLEANT position error.

\section{Distance input error}

The same observations can be made for an input error in the source-to-array distance. A slight bias may occur on the source position estimate at low frequency for MSA-PSF (Fig. 9a), at high frequency for CLEANT (Fig. 9b). The dispersion on the localization results is shown for both distance error scenarios $(20 \mathrm{~cm}$ nearer and farther $)$ for 
MSA-PSF (Fig. 9a), but not for CLEANT since negligible.

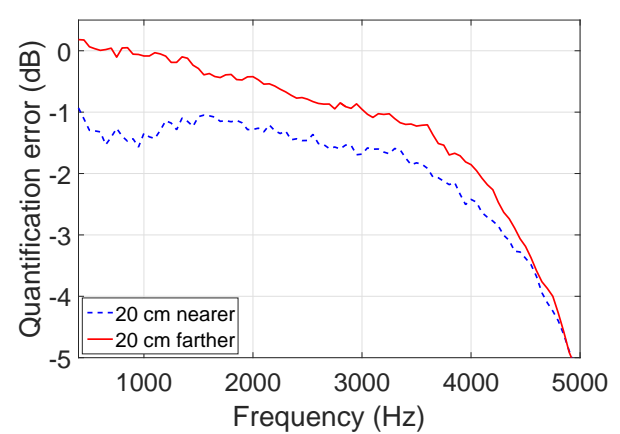

(a)

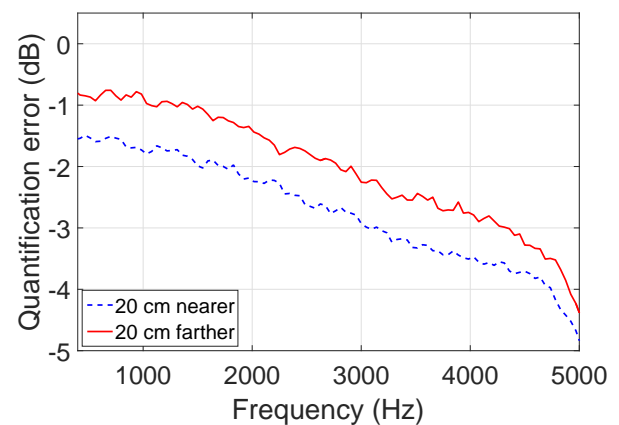

(b)

Figure 10: Averaged error on the quantification of a moving source with a wrong source-to-array distance - (a) MSA-PSF quantification error; (b) CLEANT quantification error.

If a source is not located in the grid plane investigated but at a smaller or larger distance, its quantification is highly impacted (Fig. 10). CLEANT is the most sensitive method in that case (Fig. 10b compared to Fig. 8b) and shows a lesser performance than MSA-PSF, except at higher frequencies. The standard deviation is unchanged regardless of the scenario. The figure is not included here, since similar to Fig. 5c.

\section{Experimental application of the method: case of a pendulum}

This section presents an academic experimental set-up used to test the method and compare it to MSA-PSF in real conditions, with also moving source conventional beamforming (CBF-TD) as a reference. In order to carry out a scaled experiment of a vehicle in pass-by condition, involving sources in linear motion at an approximately constant speed, a pendulum with a long rod carrying a sound device has been used. The circular movement can be locally approximated by a linear movement with the condition that the monitoring area is small with respect to the rod length. The parameters of the experiment are described below.

\subsection{Experimental parameters and configurations}

The pendulum is made with a three meter-long rigid rod that revolves around an axis supported by a weighted structure (Fig. 11). An optical system is used to get information on kinematics (position, speed). The microphone array has 30 microphones, regularly spaced by $2 \mathrm{~cm}$. Measurement is in direct proximity of the pendulum position at rest. Thus, the horizontal array is centered on this reference position and located at the height of the rod end, which supports the sources. A microphone, providing a reference signal for further research, is also fixed to the rod end and placed in direct proximity of one of the sources.

The sound device, located at $0.5 \mathrm{~m}$ from the array, is a smartphone equipped with two loudspeakers $128 \mathrm{~mm}$ apart, which has several advantages for this experiment. First, the signals are amplified inside the phone, so there is no need for a cable which would be impractical. Second, the two loudspeakers are independent and can play two different signals at the same time: two tone noises at different frequencies, or two uncorrelated white noises for instance. The last advantage of the device is its small dimensions which make it easy to fix at the rod end, and its lightness with no risk to modify the movement. Finally, acoustic panels have been placed on the floor between the pendulum and the array to minimize ground reflections.

This experiment was carried out as a scaled version of a road vehicle passing by an array of microphones, as close as possible to the typical parameters of this particular context (as studied in section 3). There is a scale factor of 7-8 on the array length and the source-to-array distance between the simulated and the experimental cases, a factor 4 in the speed considered (with respect to the $50 \mathrm{~km} \cdot \mathrm{h}^{-1}$ simulated case) and finally a wide range factor 2 to 8 between lowest frequencies and highest frequencies considered. Not every parameter could be at the exact same scale, but they were chosen to fit as best as possible to this experiment. 


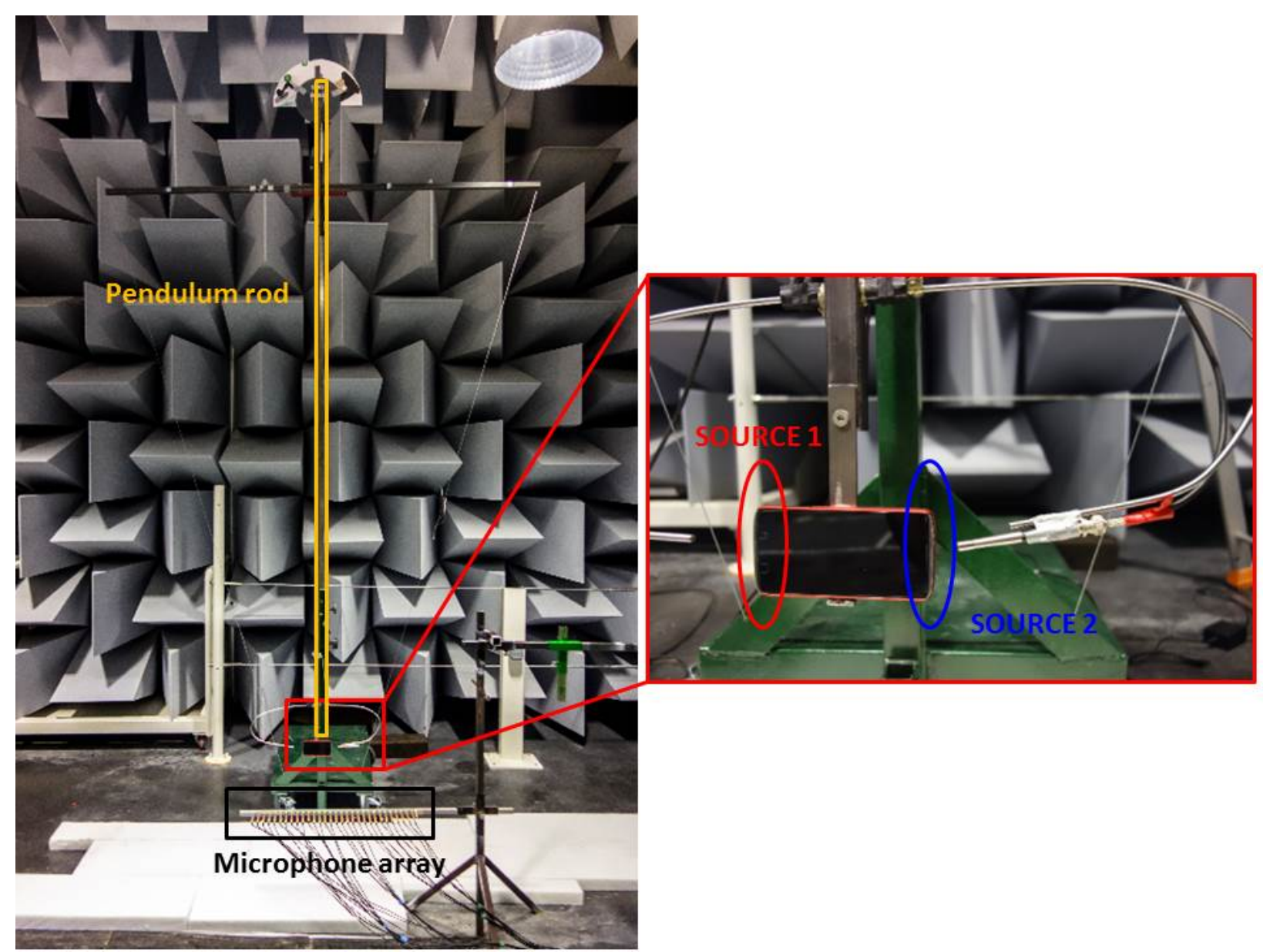

Figure 11: Overview of the experimental set-up.

The test procedure consisted in dropping the pendulum without initial speed from a variable height. Two distinct heights were chosen to obtain movements with various speeds. Two types of source signals were used : i) a tone at $6 \mathrm{kHz}$ on one speaker and another at $8 \mathrm{kHz}$ on the other one; ii) two uncorrelated white noises, one per speaker. Note that the wide band noises emitted by the device are not actual white noises though, because of the non-flat frequency response of the amplifier of each speaker.

In all of the measurements, the length of the snapshots for the MSA-PSF method was $20 \mathrm{~ms}$, corresponding to a frequency-step of $50 \mathrm{~Hz}$. The calculation has been made and averaged over 21 snapshots with a $60 \%$ overlap, which corresponds to a global signal duration of $200 \mathrm{~ms}$. Both CLEANT and CBF-TD have been implemented on one single snapshot which also lasted $200 \mathrm{~ms}$. Thus, the same section of the movement has been used in all three cases. For CLEANT, a gain factor of $\gamma=0.7$ has been chosen.

\subsection{Experimental results and method comparison}

\subsubsection{Static sources}

A preliminary measurement of the sources was made with the pendulum at rest to get a reference absolute position of the sources and an accurate estimate of the actual sound source levels.

A first method comparison is made for tone sound sources, with a $6 \mathrm{kHz}$ (resp. $8 \mathrm{kHz}$ ) tone on the right (resp. left) speaker. Conventional beamforming points out both sources, with slightly disymetrical sidelobe patterns (Fig. 12, dashed lines). Deconvolution results given by MSA-PSF (Fig. 12a) provide highly localized sources at the maximum beamforming positions, as well as several spurious lower level side peaks. These differences with the expected 
two-point source configuration are mainly due to minor discrepancies between model and reality: sound reflections and source directivities can be troublesome, along with the fact that the model considers only one source while the measurement involves two. The two main sources are actually spread over two grid points, suggesting that the estimated source location is situated between two grid samples. To get the source level, the energy of both samples is summed. On the other hand, CLEANT also provides highly localized sources but without any spurious side peaks of significance (Fig. 12b). However, the two sources are located by CLEANT with a slightly insufficient gap, being $125 \mathrm{~mm}$ appart instead of $128 \mathrm{~mm}$ expected. The sources characteristics estimated are given in Table 2. The three approaches provide very close results.

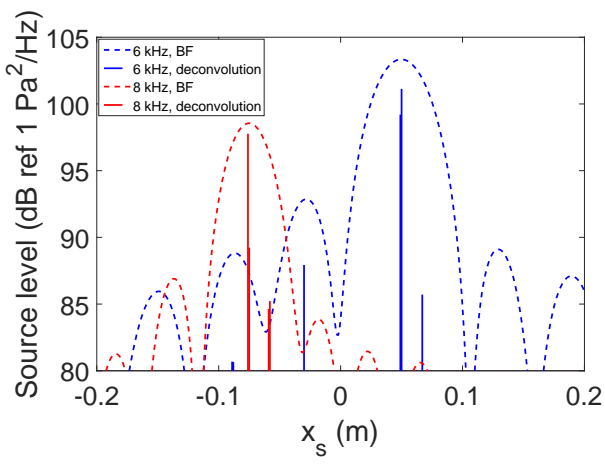

(a)

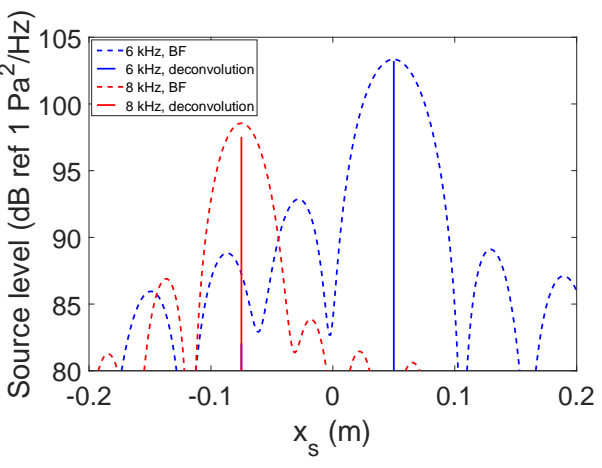

(b)

Figure 12: Deconvolution output from MSA-PSF and CLEANT for tone noise static sources - (a) MSA-PSF; (b) CLEANT - Discontinuous line: conventional beamforming output (CBF-TD).

Table 2: Source position and level estimates with static tone sources.

\begin{tabular}{cccccc}
\hline Method & \multicolumn{2}{c}{ Source 1 $(\mathbf{8} \mathbf{~ k H z})$} & \multicolumn{2}{c}{ Source 2 $(6 \mathbf{~ k H z})$} & Source gap \\
\hline & Position $(\mathrm{mm})$ & Level $(\mathrm{dB})$ & Position $(\mathrm{mm})$ & Level $(\mathrm{dB})$ & $(\mathrm{mm})$ \\
\hline CBF-TD & -77 & 99 & +50 & 103 & 127 \\
\hline MSA-PSF & -76 & 98 & +50 & 103 & 126 \\
\hline CLEANT & -75 & 98 & +50 & 103 & 125 \\
\hline
\end{tabular}

The next comparison is made with uncorrelated white noise sources. Because of the speakers gain, the emitted signal spectra are no longer constant over the frequency range. The speaker on the right has an amplification with a greater gain than the left one, in accordance with Table 2. While MSA-PSF (Fig. 13b) yields spatially narrow sources, although with a visible dispersion, CLEANT (Fig. 13c) provides highly localized sources with no ambiguity on their location: $+46 \mathrm{~mm}$ and $-78 \mathrm{~mm}$, which makes a gap of $124 \mathrm{~mm}$. The sources found by MSA-PSF are located at the estimated positions $+46 \mathrm{~mm}$ and at $-79 \mathrm{~mm}$, which makes a gap of $125 \mathrm{~mm}$ between them. The total sound level over the frequency range is $72 \mathrm{~dB}$ for the right source with CLEANT (resp. $72 \mathrm{~dB}$ with MSA-PSF), and $68 \mathrm{~dB}$ for the left source (resp. $68 \mathrm{~dB}$ ).

As a conclusion, CLEANT gives better results, with more localized sources for both tone signals and white noises and a level estimate close to the other two methods. The only concern is that the sources are found slightly closer than expected (125 $\mathrm{mm}$ for $128 \mathrm{~mm})$, but this value stays within the measurement uncertainty.

\subsubsection{Moving sources}

Only the results corresponding to the highest source speed tested are presented here, informing on the methods performance and flagrant differences in the most difficult conditions investigated. We recall that the CBF-TD applied here is adapted to moving sources, with a sweeping focus point steered on the sources (time domain).

The maximal speed of the sources in this configuration is $3.5 \mathrm{~m} \cdot \mathrm{s}^{-1}$ (speed of the pendulum when reaching the lowest position). Due to the speed measurement system uncertainty, there is an estimated $4 \%$ potential mismatch 


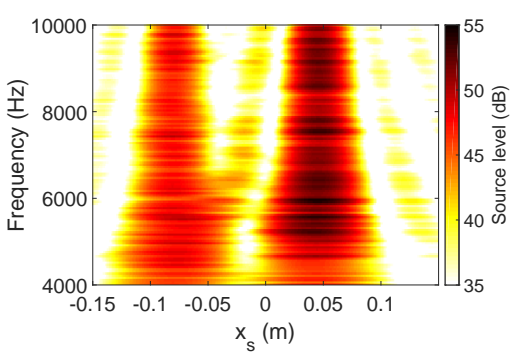

(a)

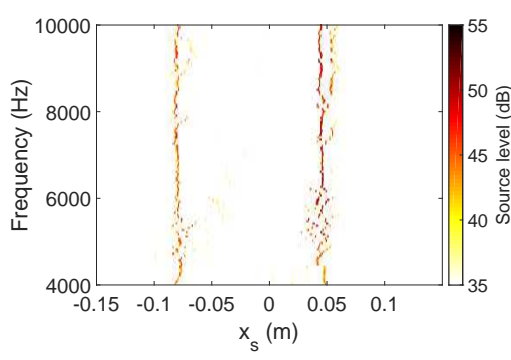

(b)

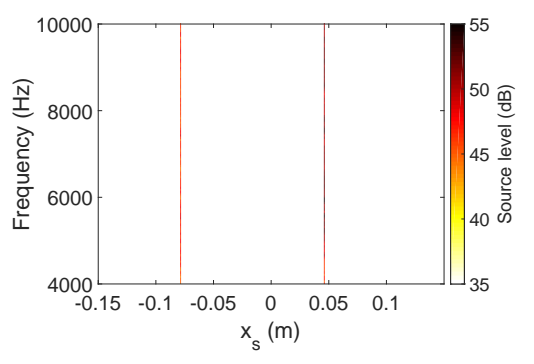

(c)

Figure 13: Comparison of the CLEANT output with the deconvolution output from MSA-PSF and CBF-TD methods for wide band noise static sources - (a) CBF-TD; (b) MSA-PSF; (c) CLEANT.

between the real sources speed and the value used in the methods, which is within the scope of the synthetic data study in section 3.3. There is also an uncertainty on the pendulum motion plane: the distance between the sources and the array may vary from $49 \mathrm{~cm}$ to $51 \mathrm{~cm}$, which represents a $2 \%$ potential mismatch, again within the scope of the study in section 3.3 where the distance error considered was of $5 \%$. The comparison is presented in the same order as in the previous subsection: first, a view of the deconvolution superposed to the beamforming output at each frequency $(6 \mathrm{kHz}$ and $8 \mathrm{kHz})$ of the tone noise sources; then the deconvolution maps in the case of white noise moving sources.

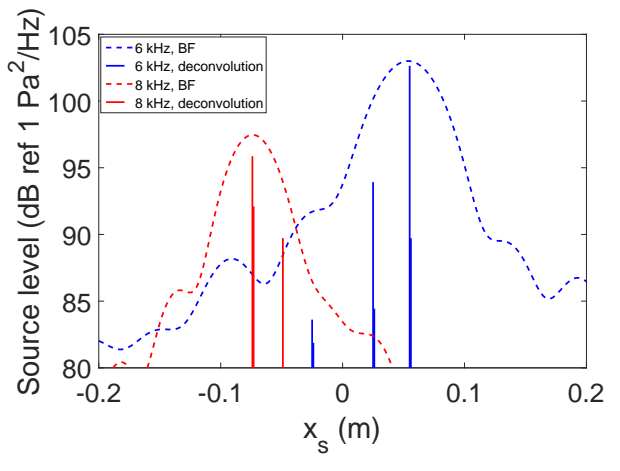

(a)

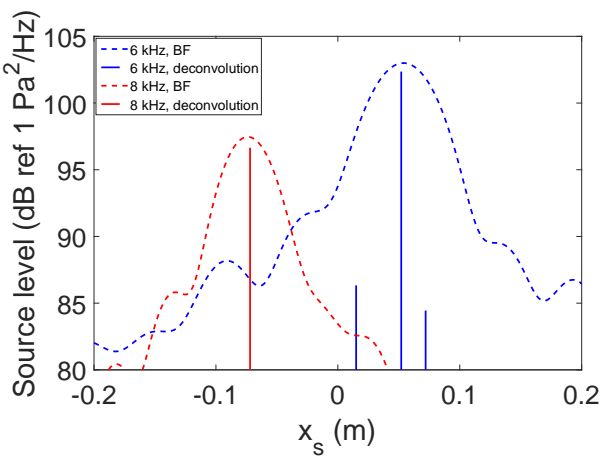

(b)

Figure 14: Deconvolution output from MSA-PSF and CLEANT for tone noise moving sources - (a) MSA-PSF; (b) CLEANT - Discontinuous line: conventional beamforming output (CBF-TD).

The beamforming output is degraded compared to the static case, with flattened sidelobes (Fig. 14, dashed lines). The MSA-PSF approach yields quite good results (Fig. 14a), however both left and right sources have a spurious side peak. Even though these side peaks have a lower level than the main ones, this might bring some ambiguity on the sources position. Though the results are degraded by the motion, the output is nonetheless good enough to locate and quantify the sources, even considering this ambiguity. Finally CLEANT yields two main sources, with spurious sidelobes for the source on the right but at noticeably lower levels (Fig. 14b). This removes the ambiguity of the position compared to MSA-PSF, but the level is slightly underestimated in this moving source context. The source characteristics estimated are given in Table 3. One can notice that with CLEANT, the gap between sources is $124 \mathrm{~mm}$, which is $1 \mathrm{~mm}$ narrower than in the static case. This effect will be found with white noise moving sources, as described below.

The beamforming map for moving white noise sources is blurrier than in the case of static sources (Fig. 15a). The MSA-PSF deconvolution map, while yielding dispersed source contributions, provides more localized positions for the two sources than the direct beamforming output (Fig. 15b) : $-75 \mathrm{~mm}$ and $+54 \mathrm{~mm}$. The gap between them is on average $129 \mathrm{~mm}$, which is hardly larger than expected. CLEANT gives a much clearer map with two well localized sources $(-73 \mathrm{~mm}$ and $+50 \mathrm{~mm}$ ) and almost no dispersion (Fig. 15c), the gap between sources being $123 \mathrm{~mm}$ 
Table 3: Source position and level estimates with moving tone sources.

\begin{tabular}{cccccc}
\hline Method & \multicolumn{2}{c}{ Source 1 (8 kHz) } & \multicolumn{2}{c}{ Source 2 $(6 \mathbf{~ k H z})$} & Source gap \\
\hline & Position $(\mathrm{mm})$ & Level $(\mathrm{dB})$ & Position $(\mathrm{mm})$ & Level $(\mathrm{dB})$ & $(\mathrm{mm})$ \\
\hline CBF-TD & -74 & 98 & +53 & 103 & 127 \\
\hline MSA-PSF & -74 & 97 & +55 & 103 & 129 \\
\hline CLEANT & -72 & 97 & +52 & 102 & 124 \\
\hline
\end{tabular}

$(1 \mathrm{~mm}$ narrower than in the static context). The total sound level over the frequency range amounts to $71 \mathrm{~dB}$ for the right source with CLEANT (resp. $71 \mathrm{~dB}$ with MSA-PSF) and to $67 \mathrm{~dB}$ for the left source (resp. $68 \mathrm{~dB}$ ). The levels are slightly underestimated compared to the static case $(72 \mathrm{~dB}$ and $68 \mathrm{~dB})$, probably because of the presence of two sources in the moving context.

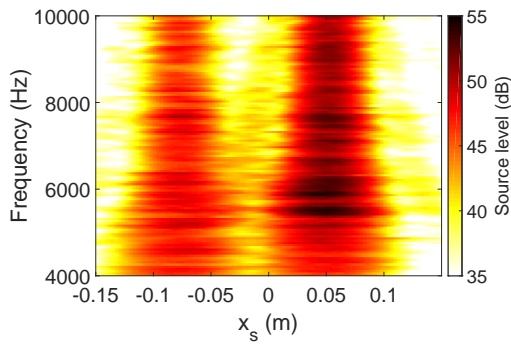

(a)

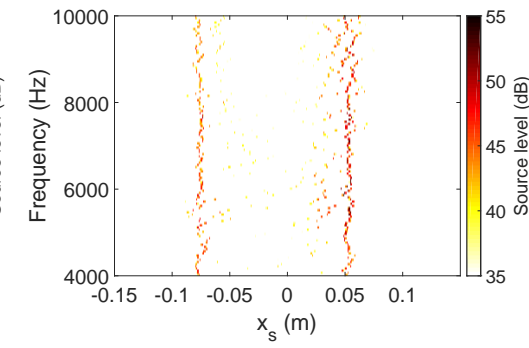

(b)

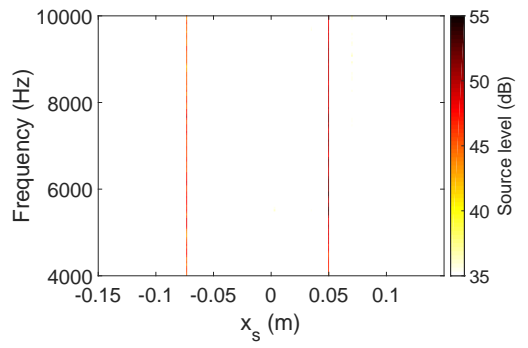

(c)

Figure 15: Comparison of the CLEANT output with the deconvolution output from MSA-PSF and CBF-TD methods for wide band noise moving sources - (a) CBF-TD; (b) MSA-PSF; (c) CLEANT.

This experiment with moving sources shows the improvement brought by CLEANT compared to MSA-PSF. The deconvolution map is precise and gives clear positions of the sources. The levels are slightly underestimated by $1 \mathrm{~dB}$ relatively to the static context, which remains quite acceptable.

An important point needs to be highlighted here, to bring some perspective on this comparison between CLEANT and MSA-PSF. The lack of dispersion in the CLEANT results regarding the wideband noise is due to its wideband treatment, since the method operates in the time domain. In the present case, the CLEANT approach takes advantage of the good localization capability in the high frequency range to improve the results at low frequency, assuming that source positions do not change as a function of the frequency. For a more complex case, with different source locations in different frequency bands, the CLEANT algorithm could include a bank-filtering step in order to improve the pertinence of the approach. This possibility is however let for future investigation.

In terms of computation time on a standard calculation laptop, the application of the methods to moving tone sources took $135 \mathrm{~s}$ with CBF-TD, $370 \mathrm{~s}$ with CLEANT (in 8 iterations) and only $40 \mathrm{~s}$ with MSA-PSF (because only the two emitted frequencies are selected for the calculation). But in the case of wideband sources, while CBF-TD and CLEANT operate in the same duration order of magnitude as for tone sources ( $160 \mathrm{~s}$ for CBF-TD, $400 \mathrm{~s}$ for CLEANT in 7 iterations), MSA-PSF is longer since every frequency studied must be treated separately: $1400 \mathrm{~s}$. Considering only these durations, it is clear that a method operating only in the frequency domain such as MSA-PSF is advantaged when the expected sources are single frequency sources or narrow bands. On the contrary, when exploring wide band noises, time domain methods such as CBF-TD and CLEANT have the edge since they handle all frequencies at the same time.

\section{Conclusion}

CLEANT is an original iterative time domain method adapted to the localization and quantification of moving sources. In case of moving sources, the formulation in time domain presents considerable advantages for a short duration pass-by when there is no possibility of multiple outcomes. Simulations using synthetic data showed a clear 
improvement over a reference method (MSA-PSF) in all the cases covered. They pointed out the importance of an accurate source kinematics knowledge since both methods are sensitive to input parameter errors. We also showed result degradation with the presence of a background noise, at low signal-to-noise ratios, which is again true for both methods, but CLEANT still yields better results under these conditions. Finally, this method is, as expected from an iterative algorithm, more time consuming than a conventional beamforming but in exchange for greatly improved accuracy.

The experimental results are encouraging for both tone sources and white noise sources, giving much clearer deconvolution maps than MSA-PSF. They underline a quantification limit for two sources in motion, the level being slightly underestimated in this case. In future work, a frequency filter will be implemented in order to improve the quantification of close sources.

\section{Acknowledgments}

This work was performed within the framework of the Labex CeLyA of Universite de Lyon, operated by the French National Research Agency (ANR-10-LABX-0060/ ANR-11-IDEX-0007).

\section{References}

[1] D. H. Johnson, D. E. Dudgeon, Array signal processing: concepts and techniques, Prentice Hall, Upper Saddle River (N.J.), USA, 1993.

[2] E. Sarradj, C. Schulze, A. Zeibig, Aspects of source separation in beamforming, in: BeBeC-2006-3, 2006 , p. 7.

[3] T. F. Brooks, W. M. Humphreys, A deconvolution approach for the mapping of acoustic sources (DAMAS) determined from phased microphone arrays, Journal of Sound and Vibration 294 (45) (2006) 856-879.

[4] J. A. Högbom, Aperture Synthesis with a Non-Regular Distribution of Interferometer Baselines, Astronomy and Astrophysics Supplement Series 15 (1974) 417.

[5] B. Barsikow, Experiences with various configurations of microphone arrays used to locate sound sources on railway trains operated by the DB AG, Journal of Sound and Vibration 193 (1) (1996) 283-293.

[6] U. Michel, History of acoustic beamforming, in: BeBeC-2006-1, 2006, p. 17.

[7] H. Kook, G. B. Moebs, P. Davies, J. S. Bolton, An efficient procedure for visualizing the sound field radiated by vehicles during standardized passby tests, Journal of Sound and Vibration 233 (1) (2000) 137-156. doi:10.1006/jsvi.1999.2794.

[8] M. Pallas, R. Chatagnon, J. Lelong, Noise emission assessment of a hybrid electric mid-size truck, Applied Acoustics 76 (2014) $280-290$. doi:10.1016/j.apacoust.2013.08.012.

[9] J. A. Ballesteros, E. Sarradj, M. D. Fernández, T. Geyer, M. J. Ballesteros, Noise source identification with Beamforming in the pass-by of a car, Applied Acoustics 93 (2015) 106-119. doi:10.1016/j.apacoust.2015.01.019.

[10] T. J. Mueller (Ed.), Aeroacoustic Measurements, Experimental Fluid Mechanics, Springer-Verlag, Berlin Heidelberg, 2002.

[11] P. Sijtsma, R. Stoker, Determination of Absolute Contributions of Aircraft Noise Components Using Fly-over Array Measurements, in: American Institute of Aeronautics and Astronautics, American Institute of Aeronautics and Astronautics, 2004, p. 14. doi:10.2514/6.20042958.

[12] U. Michel, B. Barsikow, P. Böhning, M. Hellmig, Localisation of sound sources on moving vehicles with phased microphone arrays, in: Internoise 2004, 2004, p. 7.

[13] L. Brusniak, J. Underbrink, R. Stoker, Acoustic Imaging of Aircraft Noise Sources Using Large Aperture Phased Arrays, in: American Institute of Aeronautics and Astronautics, American Institute of Aeronautics and Astronautics, 2006, p. 20. doi:10.2514/6.2006-2715.

[14] M.-A. Pallas, Localisation des sources de bruit des transports guidés (localization of noise sources on guided vehicles), Instrumentation Mesure Métrologie (I2M) 2 (1-2/2002) (2002) 29.

[15] C. A. F. de Jong, J. Bosschers, H. Hasenpflug, Model Scale Measurements of Surface Ship Radiated Flow Noise, in: NAG/DAGA, 2009 , p. 5.

[16] B. Oudompheng, Array processing for the localization and the contribution of acoustic sources of a passing-by ship using a short antenna, Theses, Université Grenoble Alpes (Nov. 2015).

[17] V. Fleury, J. Bulté, Extension of deconvolution algorithms for the mapping of moving acoustic sources, The Journal of the Acoustical Society of America 129 (3) (2011) 1417-1428. doi:10.1121/1.3531939.

[18] L. Lamotte, B. Nicolas, M. Q. Pham, B. Oudompheng, A theoretical and experimental comparison of the deconvolution methods for moving sources, in: Berlin Beamforming Conference (BebeC), 2016.

[19] R. Cousson, M.-A. Pallas, Q. Leclère, M. Bérengier, Identification of acoustic moving sources in the context of a road vehicle at pass-by, 2017, p. 040012. doi:10.1121/2.0000645.

[20] T. Brooks, W. Humphreys, A Deconvolution Approach for the Mapping of Acoustic Sources (DAMAS) Determined from Phased Microphone Arrays, in: American Institute of Aeronautics and Astronautics, American Institute of Aeronautics and Astronautics, Manchester, UK, 2004, p. 18. doi:10.2514/6.2004-2954.

[21] R. P. Dougherty, Extensions of DAMAS and Benefits and Limitations of Deconvolution in Beamforming, in: American Institute of Aeronautics and Astronautics, 2005, p. 13. doi:10.2514/6.2005-2961.

[22] M. M. Boone, Design and development of a synthetic acoustic antenna for highly directionaly sound measurements, Ph.D. thesis, Delft University of Technology, oCLC: 17688534 (1987). 
[23] R. Dougherty, R. Stoker, Sidelobe suppression for phased array aeroacoustic measurements, in: American Institute of Aeronautics and Astronautics, American Institute of Aeronautics and Astronautics, 1998, p. 11. doi:10.2514/6.1998-2242.

[24] P. Sijtsma, CLEAN Based on Spatial Source Coherence, in: American Institute of Aeronautics and Astronautics, American Institute of Aeronautics and Astronautics, 2007, p. 23. doi:10.2514/6.2007-3436.

[25] P. M. Morse, K. U. Ingard, Theoretical Acoustics, Princeton University Press, Princeton, N.J, 1992.

[26] S. Guérin, C. Weckmüller, Frequency-domain reconstruction of the point-spread function for moving sources, BeBeC-2008-14.

[27] D. Döbler, R. Schröder, Contrast improvement of acoustic maps by successive deletion of the main sources, in: BeBeC-2010-19, 2010, p. 6.

[28] D. Döbler, R. Schröder, G. Heilmann, Successive deletion of main sources in acoustic maps working in the time domain, in: Internoise 2010, 2010 , p. 8

[29] D. Döbler, D. R. Schröder, Contrast improvement and sound reconstruction of quiet sound sources using a high dynamic range algorithm, in: BeBeC-2012-14, 2012, p. 11.

[30] L. Lamotte, S. Paillasseur, K. Janssens, J. Lanslots, Enhancing Sound Source Localization with Noise Separation Methods, in: Proceedings of the Acoustics 2012 Nantes Conference, 2012, p. 8.

[31] E. Cardenas Cabada, Q. Leclre, J. Antoni, N. Hamzaoui, Fault detection in rotating machines with beamforming: Spatial visualization of diagnosis features, Mechanical Systems and Signal Processing 97 (2017) 33-43.

[32] R. Leiba, F. Ollivier, R. Marchiano, N. Misdariis, J. Marchal, Urban acoustic imaging : from measurement to the soundscape perception evaluation, in: Internoise 2016, 2016, p. 8.

[33] F. MENG, B. MASIERO, M. VORLAENDER, Compressive beamforming for moving sound source auralization, in: Internoise 2016, Hamburg, Germany, 2016, p. 7. 